\title{
ORIGINAL ARTICLE \\ Influence of neurological lesion level on heart rate variability and fatigue in adults with spinal cord injury
}

\author{
D Rodrigues ${ }^{1}, \mathrm{Y} \operatorname{Tran}^{1,2}, \mathrm{R}$ Guest $^{1}$, J Middleton ${ }^{1}$ and A Craig ${ }^{1}$
}

\begin{abstract}
Study design: Group cohort design.
Objectives: To determine the influence of spinal cord injury (SCI) and neurological level on heart rate variability (HRV) and associations with fatigue.

Setting: SCl rehabilitation outpatient and community settings in New South Wales, Australia.

Methods: Participants included 45 adults with SCl living in the community and 44 able-bodied controls. Socio-demographic, neurological injury, psychological, HRV and eye blink variables were assessed. Multivariate analysis of variance and post hoc protected $t$-tests were used to determine differences in HRV and fatigue as a function of the neurological level. Pearson's correlation analysis was used to determine the relationships between these factors.

Results: Participants with SCl had significantly reduced sympathetic activity. Those with tetraplegia had lowered sympathetic activity compared with those with paraplegia and able-bodied controls. Neither were differences in parasympathetic activity found between groups nor were there any significant differences found for the time domain or non-linear domains. Higher levels of fatigue were found in the SCl sample, and participants with tetraplegia had higher fatigue levels compared with those with paraplegia. Fatigued participants were more likely to have altered autonomic function-that is, reduced sympathetic activity.

Conclusions: Higher levels of neurological impairment in people with $\mathrm{SCl}$ are more likely to result in disordered cardiovascular control involving reduced sympathetic activity, whereas elevated fatigue was found to be associated with increased sympathetic dysfunction. Findings highlight the need to address risks associated with this dysfunction, such as improved HRV and fatigue screening for people with $\mathrm{SCl}$ and improved education on cardiovascular risk factors.
\end{abstract}

Spinal Cord (2016) 54, 292-297; doi:10.1038/sc.2015.174; published online 13 October 2015

\section{INTRODUCTION}

Spinal cord injury (SCI) involves damage to the nervous system and structures within the spinal canal (spinal cord and cauda equina), either by bruising, lacerations or severing as a result of traumatic injury or non-traumatic causes such as intervertebral disc disease, tumours, cysts and infections. ${ }^{1,2}$ Among other complications, SCI results in disordered cardiovascular control believed to be due to autonomic nervous system (ANS) imbalance and dysregulation of homeostatic mechanisms. ${ }^{2}$ Generally, dysregulation is likely to be more severe the higher and more complete the lesion, ${ }^{1,3,4}$ although inactivity associated with being wheelchair dependent may result in disordered cardiac control in lesions as low as T10.5 In cervical and high thoracic lesions, disruption of sympathetic nervous system (SNS) pathways to the heart is associated with bradycardia, orthostatic hypotension, reduced blood pressure, as well as autonomic dysreflexia and a reduced capacity to thermoregulate. ${ }^{3}$ In lesions around the T5 level and below, there is increased likelihood of problems such as uncontrolled hypertension and tachycardia. ${ }^{3,5}$ Increased cardiovascular risks in lower level lesions may be due to increased compensatory sympathetic activity. ${ }^{5}$ The mechanisms for ANS dysfunction are complex and require further investigation. ${ }^{1-5}$
Physical inactivity, metabolic syndrome and being overweight or obese increase cardiovascular risks, ${ }^{2,5}$ and arguably these problems are likely to be associated with chronic fatigue. Fatigue has been defined as a state of excessive chronic tiredness involving pervasive feelings of exhaustion and negative emotions such as anxiety and poor mood. ${ }^{6,7}$ It is the excessive and chronic nature of fatigue that distinguishes it from commonly experienced 'tiredness' related to daily physical or mental exertion, sometimes called acute fatigue. ${ }^{6}$ Chronic fatigue has been shown to be associated with problems like significantly reduced quality of life, chronic pain and mental distress. ${ }^{8,9}$ Fatigue has been found to be associated with dominant sympathetic activity in an able-bodied $(\mathrm{AB})$ sample. $^{10}$

Autonomic function changes can be measured by heart rate variability (HRV). ${ }^{10-12} \mathrm{HRV}$ is a measure of the variability of the normal-to-normal QRS complexes of the PQRST waveform of the electrocardiogram (ECG), which reflects innervation of the heart by the sympathetic and parasympathetic divisions of the ANS. It provides a measure of the relative contribution and activity of these divisions based on beat-to-beat interval variations of the heart. ${ }^{11} \mathrm{HRV}$ measures in the frequency domain have been shown to be highly reproducible in adults with SCI and therefore represent a potentially valid measure of

1John Walsh Centre for Rehabilitation Research, Kolling Institute of Medical Research, Royal North Shore Hospital, Sydney Medical School Northern, The University of Sydney, St. Leonards, New South Wales, Australia and ${ }^{2}$ Key University Centre for Health Technologies, University of Technology, Sydney, New South Wales, Australia

Correspondence: Professor A Craig, John Walsh Centre for Rehabilitation Research, Kolling Institute of Medical Research, Royal North Shore Hospital, Sydney Medical School Northern, The University of Sydney, St. Leonards, New South Wales 2650, Australia.

E-mail: a.craig@sydney.edu.au

Received 30 April 2015; revised 3 July 2015; accepted 30 August 2015; published online 13 October 2015 
ANS dysregulation in this population. ${ }^{13}$ Research employing HRV as a measure of ANS dysfunction in adults with SCI has found altered fluctuations in autonomic function in response to various activities such as tilt tests, exercise and digestion. ${ }^{14,15}$ In comparison with $\mathrm{AB}$ controls, reduced sympathetic activity was found in adults with SCI, with no differences found for parasympathetic activity. 3,5,16 Grim et al. ${ }^{3}$ studied the influence of the neurological level with small samples of adults with tetraplegia ( $n=10$ with complete lesions; $n=10$ with incomplete lesions) and adults with paraplegia $(n=10)$ on sympathovagal balance with comparisons with a small sample of $\mathrm{AB}$ controls $(n=10)$. They employed tilt, cold pressor and isometric tests to investigate the ANS function. They found significant differences in low-frequency HRV (mostly associated with sympathetic activity) between tetraplegia, paraplegia and $\mathrm{AB}$ groups. They also found high-frequency HRV (mostly associated with parasympathetic activity) differences between the paraplegia and the $\mathrm{AB}$ groups. Recent research has also found significantly reduced sympathetic activity in adults with tetraplegia, ${ }^{4}$ whereas no HRV differences were found between adults with paraplegia (T2-T12) and $\mathrm{AB}$ controls in sympathetic activity. ${ }^{5}$

The purpose of this report is to extend the research conducted in the area of HRV and SCI by, for instance, investigating the influence of SCI, on cardiovascular function using standard and non-linear HRV measures, as well clarifying the relationship between fatigue and HRV as a function of the neurological lesion level in adults with SCI. The specific aims of this study were as follows: (i) determine differences in short-term HRV measures between adults with SCI and $\mathrm{AB}$ controls; (ii) examine these HRV differences as a function of the neurological lesion level; and (iii) determine associations between SCI, HRV and fatigue. It was hypothesised that SCI results in altered HRV patterns and that greater dysfunction in HRV would occur at higher neurological levels. It was also hypothesised that elevated fatigue will be more likely to occur in participants with SCI and that in the SCI sample fatigue will be associated with disruption to the sympathetic nervous system.

Table 1 Injury characteristics of spinal cord injury participants $(n=45)$

\begin{tabular}{|c|c|c|c|c|}
\hline & & \multicolumn{2}{|c|}{ Frequency } & Percentage \\
\hline \multicolumn{5}{|c|}{ Level of injury } \\
\hline \multicolumn{2}{|l|}{ Cervical } & \multicolumn{2}{|c|}{21} & 46.7 \\
\hline \multicolumn{2}{|l|}{ Thoracic } & \multicolumn{2}{|c|}{20} & 44.4 \\
\hline \multicolumn{2}{|c|}{ Lumbar/Sacra } & \multicolumn{2}{|c|}{4} & 8.9 \\
\hline \multicolumn{5}{|c|}{ Completeness } \\
\hline \multicolumn{5}{|c|}{ Tetraplegia } \\
\hline Comp & & \multicolumn{2}{|c|}{7} & 15.6 \\
\hline Incom & & \multicolumn{2}{|c|}{14} & 31.1 \\
\hline \multicolumn{5}{|c|}{ Paraplegia } \\
\hline Comp & & \multicolumn{2}{|c|}{20} & 44.4 \\
\hline Incom & & \multicolumn{2}{|c|}{4} & 8.9 \\
\hline \multicolumn{5}{|c|}{ Severity of injury } \\
\hline & & $C 1-C 4$ & $C 5-C 8$ & T1-S5 \\
\hline \multicolumn{2}{|c|}{ Number of patients } & 13 & 8 & 24 \\
\hline ASIA & A & 3 & 4 & 20 \\
\hline ASIA & B & 1 & 1 & - \\
\hline ASIA & C & 5 & 2 & - \\
\hline ASIA & $\mathrm{D}$ & 3 & 0 & - \\
\hline Unknow & & 1 & 1 & 4 \\
\hline
\end{tabular}

Abbreviation: ASIA, American Spinal Injury Association.

\section{MATERIALS AND METHODS}

\section{Study participants}

Participants included 45 adults with a SCI (mean age $=50.4$ years, s.d. $=18 ; 38$ males, 7 females) and $44 \mathrm{AB}$ adults (mean age $=48.9$ years, s.d. $=19 ; 37$ males, 7 females). All SCI participants were recruited after discharge from inpatient rehabilitation, and sources of recruitment included outpatients at a SCI rehabilitation unit in Sydney, or from contacts of people with SCI living in the community. Mean age at injury was 40.0 years (s.d. $=18.6$, median $=37.6$, range $=17-77$ ), and mean time since injury was 10.4 years (s.d. $=12.9$, median $=2.5$, range $=0.6-47$ ). The study was advertised by placing brochures in the outpatient clinic or by placing advertisements in SCI self-help newsletters. Inclusion criteria for the participants with SCI consisted of the following: (i) aged between 18 and 80 years; (ii) understand instructions in English; (iii) sustained an acute SCI; and (iv) at least 6 months post injury from inpatient rehabilitation. As the purpose of the study was to investigate HRV in people with SCI and its associations with fatigue, $\mathrm{SCI}$ and $\mathrm{AB}$ participants with disorders like diabetes and cardiovascular disease were not excluded from the study. At the time of assessment, participants' medications included the following: 12 in the SCI group and 12 in the $\mathrm{AB}$ group were taking cardiac medications including ace inhibitors, angiotensin II, anti-platelet, beta blockers and calcium channel blockers. One participant in the SCI group and two in the $\mathrm{AB}$ group were taking anti-diabetic medication. Four in the SCI group and three in the $\mathrm{AB}$ group were taking statin medication. The most commonly prescribed medications were muscle relaxants $(n=24)$ and anti-convulsants $(n=19)$, which were used only within the SCI group. Inclusion criteria for the $\mathrm{AB}$ controls included the first two conditions for the SCI sample, as well as (iii) no history of neurological injury and (iv) no evidence of a current severe psychiatric disorder such as a bipolar disorder or psychoses as determined by a structured psychiatric interview. Injury characteristics are shown in Table 1. Approximately $58 \%$ of the $\mathrm{SCI}$ and $66 \%$ of the $\mathrm{AB}$ participants had a diploma, trade or tertiary level education. There were no significant differences in the level of education between the groups $(P=0.32)$. Falls were the most common cause of SCI $(42.2 \%)$ followed by a motor vehicle crash (30.4\%). Completeness of the lesion was assessed by a medical specialist based on International Standards for Neurological Classification of SCI (http://ais.emsci.org/). SCI participants received a $\$ 50$ gift voucher to compensate for their time and effort while $\mathrm{AB}$ participants were not remunerated.

\section{Research ethics}

Full compliance with the Code of Ethics of the World Medical Association occurred, and research ethics approval was granted by the local institutional research ethics committee. All institutional regulations concerning the ethical use of human volunteers were followed during this research. Written consent was obtained prior to participation in the study.

\section{Study design and procedure}

A group cohort design was employed. The study involved all participants completing a 40-min fatigue-inducing task during which electrophysiology recording occurred. In addition, all participants completed a battery of psychosocial measures three times: the first at baseline before the fatiguing task, the second immediately after the task and the third after a 5-min recovery period. However, only the baseline measures and the HRV data from the initial 5 min of ECG recording will be presented in this paper. All participants were requested to refrain from consuming caffeinated and alcoholic beverages on the morning of the assessment. To control for circadian influences on HRV and for fatigue status, ${ }^{10}$ assessments were conducted over a 4-h period between 9 am and $1 \mathrm{pm}$. Assessment was conducted in a quiet, relaxed environment, in a semi-darkened room to induce sleep, and was conducted either in the participant's home, a health clinic or a dedicated room in a research institution. The participants with SCI were seated in their wheelchair, and $\mathrm{AB}$ participants were seated in a chair with armrests while electrophysiological assessment occurred.

\section{Assessment}

The Biosemi Active-Two System (http://www.biosemi.com) was used in this study for recording electrophysiological signals from each participant, including 
electrooculography (EOG) and ECG, from which HRV data were obtained. For the ECG, a sampling rate of $512 \mathrm{~Hz}$ was used, being the recommended sampling rate for time domain methods and the minimal recommended sampling rate for nonparametric spectral analysis. ${ }^{11,17}$ Signal processing was performed using Kubios HRV Analysis software (Version 2.0 beta 2, Department of Applied Physics, University of Kuopio, Kuopio, Finland). ${ }^{10}$ HRV analyses were performed in the time, frequency and non-linear domains.

In the HRV time domain, measures included the mean normal-to-normal intervals, the standard deviation of the N-N interval (SDNN; the variability of the heart) and the square root of the mean squared differences of successive $\mathrm{N}-\mathrm{N}$ intervals. For short-term measures, SDNN reflects overall HRV, and square root of the mean squared differences of successive N-N intervals reflects vagal (parasympathetic) outflow. ${ }^{11}$ The frequency domain of HRV methods uses the power spectral density that measures how power (or variance) distributes as a function of frequency. ${ }^{11}$ In the frequency domain, low frequency (LF) and high frequency (HF) mostly reflect sympathetic and vagal modulations of HRV, respectively., ${ }^{411}$

In the time domain, decreased variation is often associated with a shift from sympathovagal balance to sympathetic predominance, whereas in the frequency domain this is often reflected in an increase in the LF/HF ratio. The two frequency components (LF, $0.04-0.15 \mathrm{~Hz}$ and $\mathrm{HF}, 0.15-0.35 \mathrm{~Hz}$ ), expressed in absolute values $\left(\mathrm{ms}^{2}\right)$, largely represent the degree of sympathetic and parasympathetic control, respectively. ${ }^{11}$ In the non-linear domain, entropy analyses quantify the degree of regularity, or periodicity, of the physiologic time series. Periodicity of the HR data is determined by complex interactions between multiple variables influenced by many systems, including haemodynamic, electrophysiological, humoral, autonomic and central nervous system. ${ }^{11}$ In the non-linear domain, measures include approximate entropy and sample entropy. These measures of entropy are among the most suitable non-linear analyses for short-term HRV recordings. ${ }^{11}$

Recording of the baseline HRV time series was based on a 5-min sample recording per participant, which is considered sufficient to measure HRV validly. ${ }^{11}$ However, unwanted activity in the HRV, such as mechanical and central effects of breathing and the influence of dominant very low-frequency activity, can cause distortion to time and frequency analyses, leading to possible misinterpretation of the data. ${ }^{12,18,19}$ Therefore, to distinguish changes in HR that are of interest in the investigation from these sources, an advanced detrending method was used, shown not to affect HRV statistical outcomes. ${ }^{18}$ The smoothing parameter was selected so that the HRV components of interest were not significantly affected by the detrending process. However, in the time domain, detrending results in reduced values for the SDNN, LF ms2 and Total power. ${ }^{19}$ For these reasons, the SDNN values reported in this paper cannot be used for medical diagnosis, especially where consistent normative values have not been well established. ${ }^{20}$

Fatigue was assessed using a psychometric scale called the Iowa Fatigue Scale (IFS), ${ }^{21}$ as well as an electrophysiological measure of eye blink rate duration. ${ }^{22}$
The IFS was used to measure self-reported fatigue and is composed of 11 Likert items covering 4 subscales: cognitive, fatigue, energy and productivity. ${ }^{21}$ The purpose of using the IFS was to determine the impact of a SCI on subjective fatigue levels, as well as determine whether associations exist between fatigue and HRV. To complete the IFS, participants were rated on a five-point scale of 1 'not at all' to 5 'extremely' to each item-for example, 'I feel worn out' and 'I have trouble concentrating.' The IFS has demonstrated homogeneity and internal consistency with Cronbach's coefficients over 0.90. Correlations between the four subscales ranged from 0.49 to 0.66 . An IFS total score of around 30 or higher indicates severe fatigue. ${ }^{21}$

Fatigue was also assessed by eye blink duration, provided by EOG, which has been shown to provide a sensitive physiological measure of fatigue. ${ }^{6,22,23}$ Eye blink duration was counted and measured from the unfiltered EOG raw signal at $500 \mathrm{mV}$ in 2-s intervals on a low-pass filter. For the purposes of this study, eye blink duration included both the closing and reopening times. For all participants, three cutoff times were noted and recorded: those under $300 \mathrm{~ms}$, shown to be within normal blink closure duration; those between $300-500 \mathrm{~ms}$, shown to be long lasting spontaneous blink rate, and those above $500 \mathrm{~ms}$, which included eyes closed, believed to be associated with fatigue. ${ }^{10,22}$ Baseline EOG was taken from the second minute of the 5-min baseline measure.

\section{Statistical methods}

Using Lilliefors $P$-values for the Kolmogorov-Smirnov tests, variables not normally distributed were logarithmically transformed. Consequently, all HRV variables were logarithmically transformed, except mean $\mathrm{R}-\mathrm{R}$ and sample entropy. Multivariate analysis of variance was performed to determine whether significant differences existed among the subgroups across all HRV and fatigue domains. If the multivariate analysis of variance was significant, post hoc Bonferroni protected $t$-tests were used to determine where differences existed. To determine the influence of the neurological level on HRV, the SCI group was further divided into those with tetraplegia and paraplegia, with comparisons with the $\mathrm{AB}$ group. On the basis of prior work on the differences in psychological (for example, fatigue) and physiological measures (for esample, HRV) between people with SCI and AB controls, ${ }^{7,10}$ a medium effect size was assumed (Cohen's $d$ ), and, with a sample size of 45 , 'a priori' statistical power was estimated to range between 60 and $70 \%$. Partial eta-squared $\left(\eta^{2}\right)$ effect size values are provided as an estimate of the size of the difference among the groups. A partial $\eta^{2}$ of around 0.03 is considered small, 0.13 is considered a medium difference and over 0.2 is considered a large and a substantial difference. ${ }^{24}$ Post hoc or retrospective statistical power of the tests is also provided. Pearson's correlation analyses were also conducted to examine associations between self-reported fatigue, eye blink duration times and HRV. All analyses were performed using Statistica Software (Version 12, Statsoft http://www.statsoft.com).

Table 2 Comparison of mean (s.d.) differences between HRV measures for the $\mathrm{SCl}$ and $\mathrm{AB}$ controls

\begin{tabular}{|c|c|c|c|c|c|c|}
\hline \multirow[t]{2}{*}{ Variable } & \multicolumn{2}{|c|}{ SCl group } & \multicolumn{2}{|c|}{$A B$ controls } & \multirow[t]{2}{*}{$\mathrm{F}$} & \multirow[t]{2}{*}{$\eta^{2}$} \\
\hline & Mean(s.d.) & $95 \% \mathrm{Cl}$ & Mean (s.d.) & $95 \% \mathrm{Cl}$ & & \\
\hline Mean NN & 979.04 (171.7) & $142.1-216.8$ & $936.32(159.2)$ & $131.5-201.7$ & 0.04 & 0.00 \\
\hline SDNN (In) & $29.38(0.5)$ & $12.6-19.2$ & $36.42(23.1)$ & $19.1-29.2$ & 1.67 & 0.02 \\
\hline RMSSD (In) & $29.33(20.5)$ & $16.9-25.8$ & $37.26(33.5)$ & $27.7-42.5$ & 0.77 & 0.01 \\
\hline Total $\mathrm{ms}^{2}(\mathrm{In})$ & $1035.71(1200.2)$ & $993.6-1516.1$ & $1421.13(1703.8)$ & $1407.7-2158.8$ & 0.92 & 0.01 \\
\hline $\mathrm{LF} / \mathrm{HF}(\operatorname{In})^{*}$ & $1.97(1.9)$ & $1.6-2.5$ & $2.95(4.5)$ & $3.7-5.7$ & 4.23 & 0.05 \\
\hline ApEn (In) & $1.05(0.1)$ & $0.1-0.2$ & $1.06(0.1)$ & $0.08-0.09$ & 0.26 & 0.00 \\
\hline SampEn & $1.40(0.3)$ & $0.3-0.4$ & $1.44(0.3)$ & $0.2-0.4$ & 0.52 & 0.01 \\
\hline
\end{tabular}

Abbreviations: AB, able bodied; ApEn, approximate entropy; Cl, confidence interval; HF, high-frequency band; HR, heart rate; HRV, HR variability; LF, low-frequency band; LF/HF, ratio between low-frequency and high-frequency power; LN, natural logarithm; NN, mean of the RR interval; RMSSD, root mean squared of the difference of successive RR intervals; SCI, spinal cord injury;

*Significant difference at $P<0.05$. 
Table 3 Comparison of mean (s.d.) HRV measures between tetraplegia, paraplegia and AB groups

\begin{tabular}{|c|c|c|c|c|c|c|c|c|c|}
\hline \multirow{2}{*}{ Mean RR } & \multicolumn{3}{|c|}{ Tetraplegia $(n=21)$} & \multicolumn{3}{|c|}{ Paraplegia $(n=24)$} & \multicolumn{3}{|c|}{$A B(n=44)$} \\
\hline & $\frac{\text { Mean (s.d.) }}{959.75 \text { (197.9) }}$ & \multicolumn{2}{|c|}{$95 \% \mathrm{Cl}$} & $\begin{array}{c}\text { Mean (s.d.) } \\
902.17(143.8)\end{array}$ & \multicolumn{2}{|c|}{$95 \% \mathrm{Cl}$} & $\begin{array}{c}\text { Mean (s.d.) } \\
936.32(159.2)\end{array}$ & \multicolumn{2}{|c|}{$95 \% \mathrm{Cl}$} \\
\hline STD RR & $27.10(15.8)$ & 12.1 & 22.8 & $31.38(14.6)$ & 11.4 & 20.5 & $36.42(23.1)$ & 19.1 & 29.2 \\
\hline Mean HR & $65.51(14.9)$ & 11.5 & 21.6 & $68.37(11.1)$ & 8.6 & 15.6 & $66.38(12.6)$ & 10.4 & 16.0 \\
\hline STD HR & $2.28(0.8)$ & 0.6 & 1.2 & $3.03(1.3)$ & 0.9 & 1.8 & $3.40(3.4)$ & 2.8 & 4.3 \\
\hline $\mathrm{HF}\left(\mathrm{ms}^{2}\right)$ & $518.67(876.8)$ & 670.8 & 1266.2 & 362.98 (551.9) & 428.9 & 774.2 & $512.61(723.3)$ & 597.6 & 916.5 \\
\hline Total $\left(\mathrm{ms}^{2}\right)$ & $940.24(1159.9)$ & 887.4 & 1675.1 & $1119.25(1253.1)$ & 973.9 & 1757.8 & $1421.13(1703.8)$ & 1407.7 & 2158.8 \\
\hline $\mathrm{LF} / \mathrm{HF}^{\mathrm{b}, \mathrm{c}}$ & $1.43(1.3)$ & 1.0 & 1.9 & $2.45(2.3)$ & 1.8 & 3.2 & $2.95(4.5)$ & 3.7 & 5.7 \\
\hline ApEn-1 & $1.06(0.1)$ & 0.1 & 0.2 & $1.05(0.1)$ & 0.1 & 0.2 & $1.06(0.1)$ & 0.1 & 0.1 \\
\hline SampEn-1 & $1.43(0.3)$ & 0.2 & 0.4 & $1.37(0.3)$ & 0.2 & 0.4 & $1.44(0.3)$ & 0.2 & 0.4 \\
\hline
\end{tabular}

Abbreviations: AB, able bodied; ApEn, approximate entropy; $\mathrm{Cl}$, confidence interval; HF, high-frequency band; HR, heart rate; HRV, HR variability; LF, low-frequency band; LF/HF, ratio between lowfrequency and high-frequency power; RMSSD, root mean squared of the difference of successive RR intervals; SampEn, sample entropy.

The data are presented as mean and s.d. of the raw scores.

Analyses were based on log-transformed data using natural logarithm.

a $P=0.014$ between tetraplegia and $A B$ in LF power.

${ }^{\mathrm{b}} P=0.006$ between tetraplegia and $\mathrm{AB}$ in LF/HF.

${ }^{C} P=0.047$ between paraplegia and $A B$ in LF/HF.

\section{RESULTS}

\section{HRV differences as a function of SCI}

HRV measures for the two groups are presented in Table 2. The SCI group was found to have significantly different HRV scores when compared with the $\mathrm{AB}$ group: Wilk's lambda $=0.70, F_{10,78}=3.29$, $P=0.001, \eta^{2}=0.30$, power $=98 \%$. Bonferroni's post hoc analyses showed that differences were in the frequency domain-that is, the $\mathrm{LF} \mathrm{ms}^{2}$ and $\mathrm{LF} / \mathrm{HF}$ ratio $(P<0.05)$. The SCI group had lower $\mathrm{LF} \mathrm{ms}^{2}$ and LF/HF scores. No significant differences were found between the two groups for the time and non-linear domains.

\section{HRV differences as a function of the neurological level}

Table 3 shows comparisons of HRV variables for the time, frequency and non-linear domains for the tetraplegia, paraplegia and $\mathrm{AB}$ groups. Multivariate analyses showed no differences in the HRV time and non-linear domains for the SCI subgroups and the AB group (Wilk's Lambda $=0.86, F_{10,164}=1.33, P=0.22, \eta^{2}=0.07$, power $=66 \%$; Wilk's Lambda $=0.98, \mathrm{~F}_{4170}=0.32, P=0.86, \eta^{2}=0.01$, power $=12 \%$, respectively). However, in the HRV frequency domain, there were significant differences among the tetraplegia, paraplegia and $\mathrm{AB}$ groups: Wilk's Lambda $=0.76, F_{8166}=2.98, P=0.004, \eta^{2}=0.13$, power $=95 \%$. Bonferroni's post hoc analyses indicated that differences occurred between the tetraplegia and the $\mathrm{AB}$ group in the $\mathrm{LF} \mathrm{ms}^{2}(P=0.014)$, between the tetraplegia and the $\mathrm{AB}$ group in the $\mathrm{LF} / \mathrm{HF}$ ratio $(P=0.006)$ and the paraplegia and the $\mathrm{AB}$ group in the $\mathrm{LF} / \mathrm{HF}$ ratio $(P=0.047)$. There were no significant differences found among the groups in the HF measures.

\section{Fatigue and eye blink duration as a function of the neurological} level

Table 4 shows descriptive statistics for the IFS scores and eye blink duration ( $<300 \mathrm{~ms}, 300 \mathrm{~ms}-500 \mathrm{~ms},>500 \mathrm{~ms}$ ) as a function of tetraplegia, paraplegia and the $\mathrm{AB}$ groups. Using the IFS cutoff score of 30 and above for an indication of severe fatigue, results suggest that $52.4 \%$ ( $n=11$ of 21$)$ of the tetraplegia group was experiencing severe fatigue levels when assessed at baseline (mean $=34.18$; s.d. $=4$ ), compared with $33.3 \% \quad(n=8$ of 24$)$ of the paraplegia group (mean 34.75 ; s.d. $=5)$ and $18 \%(n=8$ of 44$)$ of the $\mathrm{AB}$ group (mean $=34.25$; s.d. $=5.33)$. Similar rates of severe fatigue were found when using the eye blink duration rate $>500 \mathrm{~ms}$ as an indicator of fatigue, with $52.4 \%(n=11 / 21), 41.6 \%(n=10 / 24)$ and $11.4 \%$ ( $n=5 / 44)$ of the tetraplegia, paraplegia and $\mathrm{AB}$ groups, respectively, categorised as having elevated levels of fatigue.

Multivariate analysis of variance conducted for differences between groups for all IFS and eye blink duration indicated significant differences: Wilk's Lambda $=0.73, F_{14,160}=1.95, P=0.025, \eta^{2}=0.15$, power $=93 \%$. Univariate analyses showed that these differences were in IFS Total, Energy and Productivity domains, as well as eye blink duration $>500 \mathrm{~ms}$. Post hoc Bonferroni analyses showed that these differences occurred between the tetraplegia and the $\mathrm{AB}$ groups for the IFS total score $(P=0.016)$, energy $(P=0.026)$ and productivity $(P=0.001)$ and between paraplegia and $\mathrm{AB}$ in the $>500 \mathrm{~ms}$ eye blink duration subgroup $(P=0.034)$. A trend occurred for a difference in $>500$ ms eye blink duration between tetraplegia and the $\mathrm{AB}$ group. Overall, the tetraplegia group had higher fatigue scores compared with the paraplegia group and significantly higher fatigue scores than the $\mathrm{AB}$ group. $\mathrm{A}$ trend existed for the paraplegia group to have higher fatigue scores compared with the $\mathrm{AB}$ group.

\section{Associations between socio-demographic, HRV and fatigue measures}

For the SCI group, no socio-demographic variables significantly correlated with any fatigue or HRV variable. Table 5 shows Pearson's correlation results for associations between IFS scores, eye blink duration and LF and LF/HF measures for the SCI group. All IFS measures significantly and negatively correlated with LF power $(P<0.05)$, except for the IFS cognitive domain. Only the IFS total $(P<0.05)$ and productivity $(P<0.01)$ scores significantly and negatively correlated with the LF/HF ratio. High self-reported fatigue was associated with low levels of sympathetic activity. For eye blink duration, no significant relationships were found. No significant relationships were found between IFS or eye blink durations with $\mathrm{LF}$ power or $\mathrm{LF} / \mathrm{HF}$ ratios for the $\mathrm{AB}$ group. 
Table 4 Comparison of mean (s.d.) of IFS scores and eye blink duration between the tetraplegia, paraplegia and the AB groups

\begin{tabular}{|c|c|c|c|c|c|c|}
\hline & \multicolumn{2}{|c|}{ Tetraplegia } & \multicolumn{2}{|c|}{ Paraplegia } & \multicolumn{2}{|c|}{$A B$} \\
\hline & Mean (s.d.) & $95 \% \mathrm{Cl}$ & Mean (s.d.) & $95 \% \mathrm{Cl}$ & Mean (s.d.) & $95 \% \mathrm{Cl}$ \\
\hline IFS cognitive & $9.29(3.2)$ & $2.5-4.6$ & $8.33(2.9)$ & $2.2-4.0$ & $8.25(2.8)$ & $2.3-3.5$ \\
\hline IFS fatigue & 4.14 (1.5) & $1.2-2.2$ & 3.79 (1.9) & $1.5-2.6$ & $3.30(1.4)$ & $1.1-1.7$ \\
\hline IFS energy ${ }^{b}$ & $10.05(2.6)$ & $1.9-3.7$ & $8.63(3.0)$ & $2.4-4.3$ & $8.20(2.3)$ & $1.9-2.9$ \\
\hline EBD (300-500 ms) & $2.43(3.1)$ & $2.4-4.5$ & $2.42(3.3)$ & $2.5-4.6$ & $1.27(2.1)$ & $1.7-2.6$ \\
\hline $\operatorname{EBD}(>500 \mathrm{~ms})^{\mathrm{d}}$ & $0.90(1.1)$ & $0.8-1.6$ & $0.96(1.6)$ & $1.3-2.3$ & $0.23(0.7)$ & $0.6-0.9$ \\
\hline
\end{tabular}

Abbreviations: AB, able bodied; $\mathrm{Cl}$, confidence interval; EBD, eye blink duration; IFS, lowa Fatigue Scale.

Data are presented as mean and standard deviation (s.d.).

a $P=0.016$ between tetraplegia and $A B$.

${ }^{\mathrm{b}} P=0.026$ between tetraplegia and $\mathrm{AB}$.

${ }^{c} P=0.001$ between tetraplegia and $A B$.

$\mathrm{d} P=0.034$ between paraplegia and $A B$.

Table 5 Pearson's correlation coefficients between the fatigue-related variables (IFS and eye blink duration) and LF and LF/HF ratio HRV factors for the $\mathrm{SCl}$ group

\begin{tabular}{lcc}
\hline Fatigue variables & $L F m s^{2}(I n)$ & $L F / H F(I n)$ \\
\hline IFS total & $-0.37^{*}$ & $-0.30^{*}$ \\
IFS cognitive & -0.23 & -0.12 \\
IFS fatigue & $-0.31^{*}$ & -0.24 \\
IFS energy & $-0.34^{*}$ & -0.25 \\
IFS productivity & $-0.37^{*}$ & $-0.45^{* *}$ \\
EBD <300 ms & 0.11 & 0.29 \\
EBD 300-500 ms & -0.09 & 0.14 \\
EBD >500 ms & -0.29 & -0.16 \\
\hline
\end{tabular}

Abbreviations: HF, high-frequency band; HRV, heart rate variability; IFS, Iowa Fatigue Scale; $L F$, low-frequency band; LF/HF: ratio between low-frequency and high-frequency power; $\mathrm{SCl}$, spinal cord injury.

Data are presented as mean and standard deviation (s.d.).

${ }^{*} P<0.05,{ }^{* *} P<0.01$.

\section{DISCUSSION}

As found in prior research, ${ }^{3-5,16}$ the SCI group was found to have autonomic dysfunction, that is, reduced sympathetic activity (reduced LF and LF/HF) compared with $\mathrm{AB}$ controls of a similar age, education and sex breakdown. Participants with tetraplegia were shown to have significantly reduced sympathetic activity when compared with the $A B$ controls, also supporting the findings of prior research, ${ }^{3,4}$ whereas a trend for the participants with paraplegia to have lowered sympathetic activity compared with the controls also occurred. No differences in $\mathrm{HF}$ (parasympathetic tone) were found between the SCI and the AB groups. In addition, no significant differences were found between the two groups in the time and non-linear domains.

Malmqvist et al. ${ }^{4}$ found significantly reduced sympathetic activity in participants with tetraplegia compared with those with paraplegia (T6-T12), with no differences found between tetraplegia and those with lesions at T1-T5. Serra-Añó et al. ${ }^{5}$ found adults with paraplegia to have altered HRV compared with $\mathrm{AB}$ controls, especially in those who were less active. These findings and those presented in this paper reinforce concerns of increased risk of cardiovascular morbidity and mortality in the SCI population, recently expressed in the research literature. ${ }^{4,5}$ In response to this increased risk, our findings suggest that HRV spectral analysis should be considered more often as a screen for fatigue related to autonomic dysregulation in the SCI population. The lack of association between the non-linear HRV data and SCI requires further examination to determine whether this type of HRV complexity analysis is worthwhile.

Elevated fatigue levels were found in the SCI sample, with more severe levels found in those with tetraplegia. These elevated selfreported fatigue levels were confirmed by eye blink duration data, whereby an eye blink duration rate of $>500 \mathrm{~ms}$ (indicating fatigue or tiredness) was more likely in those with SCI. Results suggested that just over half of those with tetraplegia and around one-third of those with paraplegia had elevated/severe levels of fatigue. Significantly reduced energy and productivity were found in those with tetraplegia compared with the $\mathrm{AB}$ controls, with non-significant trends for this to occur between those with paraplegia and the $\mathrm{AB}$ controls. Fatigue in people with SCI has been linked with increased depressive mood, increased anxiety and pain. ${ }^{7,8,25}$ Results indicate that fatigue is also associated with ANS dysfunction-that is, reduced sympathetic (LF and LF/HF) activity. The lack of association between HRV and fatigue in the $\mathrm{AB}$ participants contrasts with the association found between these variables in the SCI sample.

These findings are of concern, given risks of cardiovascular disease appear to be cumulative in the SCI population-for instance, from loss of supraspinal control of ANS function, increased risk of being overweight and lowered capacity of physical activity/exercise, as well as increased occurrence of chronic fatigue, in addition to higher risks of perceived hopelessness and low self-efficacy, depressive mood and anxiety. ${ }^{7,8,26,27}$ It will be important for SCI rehabilitation programs to address these cumulative risks through self-management education concerning relationships between cardiovascular disease and lifestyle, a greater emphasis on increasing social and physical activities, as well as the establishment of psychosocial strategies that address mood, anxiety, pain and fatigue.

Limitations of the research need discussion. First, the findings presented in this paper are preliminary, based on cross-sectional data, and therefore cannot clarify time influences or causal relationships among fatigue, HRV and neurological level. There is therefore a need for experimental or prospective research to determine how fatigue contributes to HRV in people with SCI. Second, only 5-min HRV recordings were taken. However, 5-min recording of HRV is believed sufficient for valid assessment of the ANS function, ${ }^{11}$ and the HRV results based on 5-min recordings are similar to those data in studies that used longer recordings of HRV. ${ }^{4}$ This suggests that short HRV assessment could be considered a valid and a clinically pragmatic assessment of autonomic dysregulation in people with SCI. 
Furthermore, advanced detrending analysis to remove unwanted activity was employed in the 5-min recordings, which is believed to improve the validity of the HRV data and analysis. ${ }^{18}$ Third, neither were the participants screened for cardiovascular disorders nor were levels of activity/fitness assessed in the study. As described in the Materials and methods section, and perhaps as expected, there was widespread use of cardiovascular and other medications in the sample. Therefore, neither were these participants omitted nor asked to abstain from taking medications for ethical reasons. Although it is acknowledged that medications and physical fitness have a potential influence on HRV, spectral analysis results of the HRV were similar to those in other studies where these factors were considered. ${ }^{2-4}$

In conclusion, fatigue has been found to be associated with altered HRV and therefore a potential cardiovascular risk. It is hoped these results will encourage the incorporation of 'anti-fatigue and anti-cardiovascular risk' strategies that maintain or increase tonic sympathetic outflow by encouraging, for instance, appropriate levels of exercise, the use of aids such as compression stockings, improved diet and healthy sleep behaviour, and the consistent use of selfmanagement strategies such as relaxation treatments.

\section{DATA ARCHIVING}

There were no data to deposit.

\section{CONFLICT OF INTEREST}

The authors declare no conflict of interest.

\section{ACKNOWLEDGEMENTS}

We acknowledge financial support from the Australian Rotary Health.

1 Alexander M, Biering-Sorensen F, Bodner D, Brackett N, Cardenas D, Charlifue S et al. International standards to document remaining autonomic function after spinal cord injury. Spinal Cord 2009; 47: 36-43.

2 Myers J, Lee M, Kiratli J. Cardiovascular disease in spinal cord injury: an overview of prevalence, risk, evaluation, and management. Am J Phys Med Rehabil 2007; 86: $142-152$.

3 Grimm D, De Meersman R, Almenoff P, Spungen A, Bauman W. Sympathovagal balance of the heart in subjects with spinal cord injury. Am J Physiol 1997; 272: H835.

4 Malmqvist L, Biering-Sørensen T, Bartholdy K, Krassioukov A, Welling KL, Svendsen JH et al. Assessment of autonomic function after acute spinal cord injury using heart rate variability analyses. Spinal Cord 2015; 53: 54-58.

5 Serra-Añó P, Montesinos LL, Morales J, López-Bueno1 L, Gomis M, García-Massó X et al. Heart rate variability in individuals with thoracic spinal cord injury. Spinal Cord 2015; 53: 59-63.
6 Craig A, Tran Y, Wijesuriya N, Boord P. A controlled investigation into the psychological determinants of fatigue. Biol Psychol 2006; 72: 78-87.

7 Craig A, Tran Y, Wijesuriya N, Middleton J. Fatigue and tiredness in people with spinal cord injury. J Psychosom Res 2012; 73: 205-210.

8 Rodrigues D, Tran Y, Wijesuriya N, Guest R, Middleton J, Craig A. Pain intensity and its association with negative mood states in patients with spinal cord injury. Pain Ther 2013; 2: 113-119.

9 Wijesuriya N, Tran Y, Middleton J, Craig A. The impact of fatigue on the health related quality of life in persons with spinal cord injury. Arch Phys Med Rehabil 2012; 93: 319-324.

10 Tran Y, Wijesuriya N, Tarvainen M, Karjalainen P, Craig A. The relationship between spectral changes in heart rate variability and fatigue. J Psychophysiol 2009; 23: 143-151.

11 Malik M, Bigger JT, Camm AJ, Kleiger RE, Malliani A, Moss AJ et al. Task Force of the European Society of Cardiology and the North American Society of Pacing and Electrophysiology. Heart rate variability standards of measurement, physiological interpretation, and clinical use. Eur Heart J 1996; 17: 354-381.

12 Yum MK, Kim JT, Kim HS. Increased non-stationarity of heart rate during general anaesthesia with sevoflurane or desflurane in children. Brit J Anaesth 2008; 100: 772-779.

13 Ditor DS, Kamath MV, Macdonald MJ, Bugaresti J, McCartney N, Hicks AL. Reproducibility of heart rate variability and blood pressure variability in individuals with spinal cord injury. Clin Auton Res 2005; 15: 387-393.

14 Bluvshtein V, Korczyn A, Akselrod S, Pinhas I, Gelernter I, Alexander M et al. International standards to document remaining autonomic function after spinal cord injury. Spinal Cord 2008; 47: 36-43.

15 Catz A. Hemodynamic responses to head-up tilt after spinal cord injury support a role for the mid-thoracic spinal cord in cardiovascular regulation. Spinal Cord 2011; 49: 251-256.

16 Bunten DC, Warner AL, Brunnemann SR, Segal JL. Heart rate variability is altered following spinal cord injury. Clin Autonomic Res 1998; 8: 329-334.

17 Singe D, Vinod K, Saxena S. Sampling frequency of the RR interval time series for spectral analysis of heart rate variability. J Med Eng Technol 2004; 28: 263-272.

18 Tarvainen MP, Ranta-Aho PO, Karjalainen PA. An advanced detrending method with application to HRV analysis. IEEE Trans Biomed Eng 2002; 49: 172-175.

$19 \mathrm{Li} \mathrm{L}$, Liu C, Liu C. Comparison of detrending methods in spectral analysis of heart rate variability. Res J Appl Sci Eng Technol 2011; 3: 1014-1021.

20 Nunan D, Sandercock GR, Brodie DA. A quantitative systematic review of normal values for short-term heart rate variability in health adults. Pacing Clin Electrophys 2010; 33: 1407-1417.

21 Hartz A, Bentler S, Watson D. Measuring fatigue severity in primary care patients. J Psychosom Res 2003; 54: 515-521.

22 Caffier PP, Erdmann U, Ullsperger P. Experimental evaluation of eye-blink parameters as a drowsiness measure. Eur J App Physiol 2003; 89: 319-325.

23 Schleicher R, Galley N, Briest S, Galley L. Blinks and saccades as indicators of fatigue in sleepiness warnings: looking tired? Ergonomics 2008; 51: 982-1010.

24 Cohen J. Statistical power for the social sciences. Laurence Erlbaum and Associates: Hillsdale, NJ, 1988.

25 Craig A, Tran Y, Siddall P, Wijesuriya N, Lovas J, Bartrop et al. Developing a model of associations between chronic pain, depressive mood, chronic fatigue and self-efficacy in people with spinal cord injury. J Pain 2013; 14: 911-920.

26 Hancock K, Craig AR, Tennant C, Chang E. The influence of spinal cord injury on coping styles and self-perceptions: a controlled study. Aust N Z J Psychiatry 1993; 27: 450-456.

27 Craig A, Hancock K, Chang E, Dickson H. The effectiveness of group psychological intervention in enhancing perceptions of control following spinal cord injury. Aust $N Z J$ Psychiatry 1998; 32: 112-118. 